\title{
Retraction Note to: Downregulation of microRNA-217 and microRNA-646 acts as potential predictor biomarkers in progression, metastasis, and unfavorable prognosis of human osteosarcoma
}

\author{
Afshin Taheri Azam ${ }^{1}$ - Reza Bahador ${ }^{2}$ - Hamid Hesarikia ${ }^{3}$ - Mohammadreza Shakeri ${ }^{2}$. \\ Ali Yeganeh ${ }^{4}$
}

Published online: 5 November 2016

(C) International Society of Oncology and BioMarkers (ISOBM) 2016

Retraction Note to: Tumor Biol (2016) 37:5769-5773

DOI 10.1007/s13277-015-3821-4

This article has been retracted at the request of the Editor-in-Chief, the International Society of Oncology and BioMarkers (ISOBM) and the Publisher per the Committee on Publication Ethics guidelines. The article shows evidence of irregularities in authorship during the submission process, there is strong reason to believe that the peer review process was compromised and there are similarities with the following articles which were all submitted within a close timeframe:

Masoomeh Dadpay, Mojtaba Zarea, Rahman Ghaffarzadegan Rabati, Bijan Rezakhaniha, Babak Barari, Vahid Behnod, Katayoun Ziari, Upregulation of miR-21 and downregulation of miR-494 may serve as emerging molecular biomarkers for prediagnostic samples of subjects who developed nasopharyngeal carcinoma associates with lymph

The online version of the original article can be found at http://dx.doi.org/ 10.1007/s13277-015-3821-4.

Ali Yeganeh

yeganeh471@yahoo.com

1 Department of Orthopedics Surgery, Tehran Medical Branch, Islamic Azad University, Tehran, Iran

2 Department of Orthopaedic and Trauma Surgery, Birjand University of Medical Sciences, Birjand, Iran

3 Trauma Research Center, Department of Orthopedics, Baqiyatallah Hospital, Baqiyatallah University of Medical Sciences, Tehran, Iran

4 Department of Orthopedic Surgery, Rasoul Akram Hospital, Iran University of Medical Sciences, Tehran, Iran node metastasis and poor prognosis. Tumor Biol. First Online: 21 August 2015 DOI: 10.1007/s13277-015-3905-1

Date received: 27 June 2015

Seyyed Hasan Karbasy, Afshin Taheriazam, Alireza Mirghasemi, Farnoush Sedaghati, Mohammadreza Shakeri, Emad Yahaghi, Reza Bahador, Upregulation of miR-300 and downregulation of miR-125b act as potential predictor biomarkers in progression, metastasis, and poor prognosis of osteosarcoma. Tumor Biol. First Online: 02 September 2015 DOI: 10.1007/s13277-015-4000-3

Date received: 24 July 2015

The retracted article was received: 5 July 2015 .

As such the validity of the content of this article cannot be verified. 